\title{
Hormiga argentina Linepithema humile Mayr, 1868 (Hymenoptera: Formicidae) y su rol como posible vector de contaminación microbiana en una lechería de cabras Capra hircus Linnaeus, 1758 (Artiodactyla: Bovidae)
}

\author{
Argentine ant Linepithema humile Mayr, 1868 (Hymenoptera: Formicidae) and its role as a \\ possible vector of microbial contamination in a dairy goat farm Capra hircus Linnaeus, 1758 \\ (Artiodactyla: Bovidae) \\ J Ipinza-Regla ${ }^{a^{*}}$, D González ${ }^{a}$, G Figueroab \\ a'Laboratorio de Zoología y Etología, Facultad de Ciencias Silvoagropecuarias, Universidad Mayor, Santiago, Chile. \\ bInstituto de Nutrición y Tecnología de los Alimentos, Universidad de Chile, Santiago, Chile.
}

\begin{abstract}
SUMMARY
A bacteriological study of ants was conducted. The ants were captured in a dairy goat establishment located in Metropolitan Region. 63 samples were obtained and grouped into: 21 control samples aspirated directly on the sterile filter paper, prior to the passing of ants (Control A), 21 ant samples aspirated from the filter paper (Ants Sample), after removal of the ants from the aforementioned filter paper, 21 samples were obtained after the passing of ants (Control B). A microbiological protocol specifically designed for this study was used. The methodology included sowing in nutritious, selective and differential culture media. Subsequently, and depending on the organism biochemical tests or specific tests followed in each case. In this study it was decided to investigate the presence of 6 important pathogenic agents: Staphylococcus aureus, Salmonella spp., Pseudomonas aeruginosa, Escherichia coli, Listeria monocytogenes and Campylobacter jejuni. Four potential pathogens were isolated from the Ants Samples and the Control B Samples:Staphylococcus aureus, Salmonella spp., Pseudomonas aeruginosa and Escherichia coli. Also, other microbial agents were found such as Streptococcus sp., Micrococcus sp., Escherichia vulneris, Hafnia alvei, Serratia marcencens biog.1, Ewingella americana, Providencia rettgeri, Bacillus sp., Enterobacter aglomerans, Stomatococcu ssp., Morganella morganii. Control A samples were negative to microbial growth. Ant cultures were positive in $95.23 \%$, while Control B samples showed bacterial growth in $90.47 \%$ of the cases. Linepithema humile, is capable of carrying microbial agents in a dairy goat farm.
\end{abstract}

Key words: Argentine ant, Capra hircus, microbial contamination.

\section{RESUMEN}

Se trata de establecer la acción de la hormiga argentina Linepithema humile, como potencial vector mecánico de microorganismos patógenos. Desde un plantel lechero de cabras ubicado en la comuna de Lampa, Región Metropolitana, Chile, se obtuvieron 63 muestras: 21 muestras Control A aspiradas directamente sobre el papel filtro esterilizado previo al paso de las hormigas, 21 muestras de hormigas aspiradas desde papel filtro y 21 muestras posterior al paso de las hormigas (Control B). La metodología incluyó siembras en medios de cultivos nutritivos, selectivos y diferenciales. Posteriormente y según el microorganismo a seguir se continuó con algunas pruebas bioquímicas específicas correspondientes a cada caso. En el presente estudio se decidió investigar la presencia de seis agentes patógenos importantes por su frecuencia en caso de infección en humanos: Staphylococcus aureus, Salmonella spp., Pseudomona aeruginosa, Escherichia coli, Listeria monocytogenes y Campylobacter jejuni. En los resultados de este estudio se detectaron cuatro potenciales patógenos: S. aureus, Salmonella spp., P. aeruginosa y E. coli. Además se encontraron otros agentes microbianos: Streptococcus spp., Micrococcus spp., Escherichia vulneris, Hafnia alvei, Serratia marcencens biog.1, Ewingella americana, Providencia rettgeri, Bacillus spp., Enterobacter aglomerans, Stomatococcus spp., Morganella morganii. Las muestras Control A resultaron negativas. Las muestras de hormigas fueron positivos en $95,23 \%$, en cambio, las muestras controles B presentaron desarrollo bacteriano en $90,47 \%$ de los casos, siendo ambos estadísticamente mayores al control A $(\mathrm{P} \leq 0,05)$. Se logró confirmar que $L$. humile es capaz de transportar agentes microbianos en una lechería de cabras.

Palabras clave: hormiga argentina, Capra hircus, contaminación microbiana.

\section{INTRODUCCIÓN}

La industria de la leche y sus derivados, de origen caprino, crece en forma continua ampliando y ofertando cuidados sanitarios cada vez más complejos, muchas

\footnotetext{
Aceptado: 12.03.2015.

\# FIDUM Universidad Mayor- Laboratorio de Microbiología INTA, Universidad de Chile

* joaquin.ipinza@umayor.cl
}

veces sujetos a normas de calidad que exige el mercado consumidor. Frente a esto el Médico Veterinario debe garantizar que los productos provenientes de los animales cumplan con los requisitos microbiológicos (especificados en el Reglamento Sanitario de los alimentos RSA, Ministerio de Salud), con los Límites Máximos para Residuos (LMR) y Límites Máximos para Residuos Extraños (LMRE) (establecidos por Codex Alimentarius), y de esta manera evitar que sean perjudiciales para el ser humano. 
Entre las medidas para asegurar la inocuidad es de real importancia enfocar las medidas sanitarias en el control de plagas y enfermedades. Habitualmente el control se realiza contra vectores ampliamente estudiados y por tal razón conocidos, en cuanto al rol que cumplen como vehículo de flora patógena relevante, y que no solo producen daño a nivel de producción, sino que, en algunos casos, pueden afectar la salud del hombre (Ipinza-Regla y col 1981, 1984).

En cuanto al peligro que presenta la hormiga argentina, esta no solo constituye un potencial riesgo para la salud humana, sino que también amenaza con extinguir especies de hormigas nativas (Ipinza-Regla y col 2010), además se le atribuyen daños a nivel de cultivos. Algunos estudios establecen los efectos negativos en los árboles frutales (Ripa y col 1993a ${ }^{\mathrm{a}}$ 1993 ${ }^{\mathrm{b}}$, Larraín y col 1995, Ripa y col 1998). Incluso recientes estudios acerca del efecto de la depredación de formícidos, entre ellos se estudió a la hormiga argentina frente a termitas, resultó que esta especie de formícido produjo más muertes y daños a las termitas (Osorio 2010).

Desde el punto de vista de salud pública, las hormigas han sido consideradas como insectos inofensivos, aun cuando tienen fácil acceso a lugares asépticos en hospitales e industrias de alimentos, entre otros.

La producción caprina tradicional de nuestro país tiene una orientación especialmente lechera, la que se destina a la elaboración de quesos, básicamente de tipo fresco. Sin embargo, gran parte de esa producción quesera se realiza sin cumplir con las normas de higiene estipuladas por el Reglamento Sanitario de los Alimentos del Ministerio de Salud de Chile por parte de campesinos de bajo nivel sociocultural, obteniéndose un alimento de alto riesgo sanitario (Lazo 2002).

A pesar de las medidas sanitarias para evitar las plagas de insectos u otros, en recintos pecuarios, las hormigas insistentemente se pueden observar en la mayoría de estos. Las podemos encontrar tanto en comederos como en salas de ordeña, y zonas donde se almacena el alimento. En consecuencia, se hace necesario dilucidar el riesgo que estos formícidos son capaces de presentar en planteles de producción animal.

Fuera de la escasa información de las hormigas de la especie Linepithema humile como vector de microorganismos patógenos en Chile en ambientes intrahospitalarios e industrias de alimentos (Ipinza-Regla y col 1981, 1984), no existe información de esta hormiga en relación con los animales de abasto. Por ello se propone analizar bajo las condiciones nacionales si es posible identificar que $L$. humile pudiera tener un rol en el transporte de microorganismos patógenos involucrados en el ganado caprino.

De acuerdo con lo anterior, el objetivo del presente trabajo fue obtener información acerca de la posible capacidad que tiene la hormiga argentina Linepithema humile Mayr, 1868, para transportar en forma mecánica microorganismos y verificar si ellos pueden representar un riesgo para la salud, especialmente en el rebaño caprino de un plantel lechero de explotación intensiva, ubicado en la región Metropolitana (Santiago, Chile). Además se pretendió verificar si el transporte eventual de microorganismos patógenos pudiera significar un riesgo de contaminación de los alimentos (leche y quesos) que pudieren causar infecciones o enfermedades en el consumidor humano.

\section{MATERIAL Y MÉTODOS}

Con el fin de investigar la capacidad de L. humile Mayr, 1868, para transportar en forma mecánica agentes patógenos en una lechería de cabras Capra hircus (Linnaeus, 1758) se procedió a la recolección de especies aplicando la técnica de toma de muestras similar a la descrita por Ipinza-Regla y col (1981).

Se analizaron 21 muestras por cada grupo: Grupo Control A (control del procedimiento), Grupo Experimental Hormigas (H) y Grupo Control B (control de superficie luego del paso de las hormigas), según esto se analizaron 63 muestras.

Los controles (A y B) no consideran aspirados de hormigas $(\mathrm{H})$. El control A correspondió al aspirado sobre el papel filtro previo al paso de las hormigas y la muestra control B correspondió al aspirado sobre el papel filtro posterior al paso de los insectos. Cada muestra de hormigas $(\mathrm{H})$ correspondió a la captura de 15 ejemplares (por medio de los aspiradores estériles).

El muestreo se realizó en diciembre y enero, época de mayor actividad de estos insectos sociales, en un plantel de cabras ubicado en la comuna de Lampa, sector Lo Vargas, Región Metropolitana. La dotación de animales la constituían 320 cabras, con un sistema de producción correspondiente a un tipo de explotación intensiva. La raza en estudio fue Saanen, mestiza Saanen y Alpina francesa.

Para la recolección de muestras se emplearon aspiradores (de uso común en entomología), papel filtro y una solución de glucosa al $20 \%$, todo previamente esterilizado (para este fin se utilizó autoclave a una temperatura de $121{ }^{\circ} \mathrm{C}$ durante 15 minutos; en cambio, para la solución glucosa se hizo mediante filtración).

Una vez determinado el sendero de hormigas en las horas de mayor actividad de estos insectos (Ipinza-Regla y col 1991) se precisó el lugar para colocar el papel filtro estéril. Dicho papel se instaló previo al paso de las hormigas, para verificar el grado de contaminación del medio ambiente y comprobación de que la manipulación no influye en la contaminación de las muestras.

Después, de manera de atraer la mayor cantidad de ejemplares se agregan algunas gotas de glucosa estéril sobre el papel filtro. Posteriormente se tomó la muestra de hormigas con un segundo aspirador estéril, recolectándose una cantidad de 15 hormigas en total. Por medio de suaves golpes con una bagueta estéril se ahuyentó las hormigas restantes fuera del papel. Enseguida se tomó la muestra control B aspirando sobre las zonas del papel por 
donde pasaron los insectos con un tercer aspirador estéril (Ipinza-Regla y col 1984).

Obtenidas la totalidad de las muestras se trasladaron inmediatamente al Laboratorio de Microbiología del Instituto de Nutrición y Tecnología de los Alimentos de la Universidad de Chile (INTA), para su desarrollo.

El procesamiento consistió en adicionar $10 \mathrm{~mL}$ de caldo cerebro corazón (Brain Heart $\mathrm{BH}$ ) estéril a cada frasco control A, hormigas $(\mathrm{H})$ y control $\mathrm{B}$, manteniéndose a temperatura ambiente durante 2-3 horas con el objetivo de lograr una homogenización de la muestra; al final de este lapso se utilizaron medios de preenriquecimiento para todas las muestras, excepto para Pseudomona aeruginosa la que se sembró directamente en el medio de cultivo. Al cabo de este tiempo se continuó con el protocolo diseñado especialmente para esta investigación.

Transcurridas 2-3 horas a temperatura ambiente en el caldo $\mathrm{BH}$, se extrajo $1 \mathrm{~mL}$ desde las muestras $\mathrm{H}$ y las muestras control B, contenido que se inocula a cada tubo que contienen $9 \mathrm{~mL}$ del respectivo caldo de preenriquecimiento, este se lleva a incubación durante 24 horas a $37^{\circ} \mathrm{C}$ y para el caso de Campylobacter jejuni a $42^{\circ} \mathrm{C}$ en ambiente de microaerofilia. Los caldos de preenriquecimiento ultilizados fueron: para Campylobacter, Caldo Hunt; para Staphylococcus, Caldo Soya Tripticasa (TSB); para Salmonella, Caldo Lactosado, Caldo Tetrationato (TT) y Rappaport-Vassiliadis (RV); para Listeria, Caldo Buffered Listeria Enrichment Broth (BLEB); para Escherichia coli, Caldo Enrichment Broth (EEB).

Al término de este lapso y después de haber comprobado tanto la ausencia de desarrollo microbiano en la muestra control A como desarrollo microbiano en las muestras $\mathrm{H}$ y los controles $\mathrm{B}$, se procedió a sembrar los positivos desde los caldos de enriquecimiento en los siguientes medios sólidos de cultivo: Skirrow, McConkey sorbitol, Baird Parker, Palcam y Oxford, para Campylobacter jejuni, Echerichia coli, Staphylococcus aureus, y Listeria monocytogenes, respectivamente.

En el caso de Salmonella, el inóculo se traspasa al caldo de enriquecimiento (Tetratoniato y Rappaport) por $24 \mathrm{~h}$ en baño termorregulado a $43^{\circ} \mathrm{C}$. Luego se traspasan a medios sólidos Xilosa Lisina Desoxicolato (XLD) y Hecktoen entérico (HK). En el caso de L. monocytogenes, el caldo de preenriquecimiento (BLEB) se deja 24 y $48 \mathrm{~h}$ en la estufa a $37^{\circ} \mathrm{C}$ y luego se siembra en medios sólidos (Palcam y Oxford).

La técnica bacteriológica utilizada en las placas fue por diseminación en estrías y de esta manera obtener colonias aisladas para identificarlas y describirlas según características macroscópicas (tamaño, forma, color, superficie, etc.) y microscópicas (tinción Gram).

Las cepas aisladas pertenecientes a la familia Enterobacteriaceae (Salmonella y E. coli, específicamente) fueron sometidas a tipificación serológica con antisueros específicos mono y polivalentes, según la técnica de aglutinación en lámina.
Para otras cepas aisladas de interés en este trabajo, fueron sometidas a pruebas de sensibilidad in vitro con antibióticos.

Se analizó el desarrollo bacteriano en muestras tomadas en una lechería de cabras de la Región Metropolitana, así como también el porcentaje de muestras de hormigas (H) y muestras control B con diferentes cepas bacterianas aisladas. Por último se analizó la frecuencia de presentación de microorganismo en relación con el total de cepas aisladas en 21 muestras de hormigas y la frecuencia de presentación de microorganismos con relación al total de cepas aisladas en 21 muestras control B, ambos valores expresados en porcentajes.

\section{RESULTADOS}

Las muestras controles A no presentaron desarrollo microbiano, cumpliendo el objetivo de que la primera muestra se obtuviera de un papel filtro previamente esterilizado. Los cultivos de hormigas fueron positivos en $95,23 \%$ de las muestras, en cambio los controles B presentaron desarrollo bacteriano en $90,47 \%$ de los casos, siendo ambos diferentes estadísticamente al control $(\mathrm{P} \leq$ 0,05; cuadro 1).

De las muestras de hormigas se obtuvieron microorganismos Gram positivos (G+) y otros Gram negativos (G-), siendo los $\mathrm{G}+$ los que se encontraron en mayor cantidad de muestras, a diferencia de los G- que tuvieron una frecuencia de presentación menor, pero con mayor cantidad de especies localizadas.

Algunos de los hallazgos encontrados en las muestras de hormigas se puede mencionar que el microorganismo más frecuentemente aislado fue Bacillus spp., hallándose en 13 de 21 muestras $(61,9 \%)$. Los otros dos microorganismos más asiduamente aislados corresponden a Staphylococcus spp. y bacilos Gram negativos no fermentadores, encontrándose en 11 y 9 muestras, respectivamente.

En 9,52\% de las muestras se encontró Hafnia alvei, Serratia marcencens biog. 1, Micrococcus spp. Streptococcus spp. y Morganella morganii.

Se aislaron 8 agentes microbianos, en un porcentaje de 4,76\%, es decir, en 1 de 21 muestras. Los microorganismos que se encontraron en menor frecuencia son:

Cuadro 1. Desarrollo bacteriano (expresado en porcentaje) en 63 muestras tomadas en una lechería de cabras en la Región Metropolitana.

Bacterial growth (percentage) in 63 samples from a dairy goat in the Metropolitan Region.

\begin{tabular}{lcc}
\hline Muestras & $\begin{array}{c}\text { Desarrollo } \\
\text { bacteriano }+\end{array}$ & $\%$ \\
\hline Control A & 0 & 0 \\
Hormigas & 20 & 95,23 \\
Control B & 19 & 90,47 \\
\hline
\end{tabular}


Escherichia vulneris, Escherichia coli, Ewingella americana, Pseudomona aeruginosa, Providencia rettgeri, Enterobacter aglomerans, Stomatococcus spp. y Salmonella spp.

Respecto de la cantidad de cepas aisladas en este tipo de muestras, es importante mencionar que se aislaron 59 cepas, correspondiendo a 16 especies diferentes, perteneciendo cinco de estas a G+ y once G-. Presentando un porcentaje mayor de aislamiento Staphylococcus spp., luego con un mismo porcentaje Bacillus spp., y bacilos G- no fermentadores.

Se logró demostrar que dos de los tres más altos porcentajes en su frecuencia de presentación corresponden a microorganismos $\mathrm{G}+$, y solo uno de ellos a $\mathrm{G}-$, sin embargo, ninguna de estas cepas bacterianas son parte de los seis microorganismos patógenos buscados en este estudio (figura 1).

En las muestras control B, Staphylococcus spp. fue el agente microbiano detectado en la mayor cantidad de muestras (42,86\%). A continuación, el 38,09\% de las muestras se evidenció la presencia de Bacillus spp., y el $19,04 \%$ bacilos $\mathrm{G}-$ no fermentadores.

Se logró aislar cepas de Micrococcus spp. en 3 muestras (14,28\%), Hafnia alvei y Escherichia coli en 2 muestras $(9,52 \%)$. Por último, menor prevalencia tuvieron los aislamientos de Serratia marcencens biog. 1, Morganella morganii y Staphylococcus aureus, en una sola muestra, correspondiendo al 4,76\% (figura 2).

El total de cepas aisladas de las 21 muestras procesadas corresponden a 35. De estas, 21 cepas pertenecen a microorganismos G+, entre ellas están S. aureus, Staphylococcus spp., Bacillus spp. y Micrococcus spp.; en cambio solo 10 cepas pertenecen a microorganismos $\mathrm{G}-$, como por ejemplo E. coli, Bacilos G- no fermentadores, Hafnia alvei, Serratia marcencens biog. 1, Morganella morganii.

La frecuencia de presentación más alta dentro de las cepas bacterianas G+ correspondó a 25,71\% Staphylococcus spp. y 22,85\% Bacillus spp. En las cepas bacterianas G-, bacilos $\mathrm{G}$ - no fermentadores fueron los que obtuvieron un mayor porcentaje en frecuencia de presentación $(22,85 \%)$.

En las muestras control B, las mayores frecuencias de presentación las obtuvieron Staphylococcus spp. (25,7\%), Bacillus spp. y bacilos G- no fermentadores (ambos con $22,9 \%)$. Con menor porcentaje Escherichia coli $(5,7 \%)$ y Staphylococcus aureus (2,9\%), ambos de importancia sanitaria (figura 3).

\section{DISCUSIÓN}

Con la metodología aplicada en esta investigación se logró determinar que la hormiga argentina obtenida en una lechería de cabras de la Región Metropolitana es capaz de transportar microorganismos patógenos.

Los resultados obtenidos en este estudio revelaron que los microorganismos aislados más frecuentemente coinciden en la gran mayoría con los aislados en los estudios realizados anteriormente en Chile (Ipinza-Regla y col 1981, 1984) y en Colombia (Olaya y col 2005).

Los resultados obtenidos para la hormiga argentina en Chile como vector de microorganismos patógenos, obtenidos tanto en ambientes intrahospitalarios como en fábricas de hamburguesas y confites, arrojaron algunas diferencias importantes de mencionar. En ambientes intrahospitalarios, los microorganismos que tuvieron una mayor frecuencia de presentación en muestras de hormigas fueron Bacillus

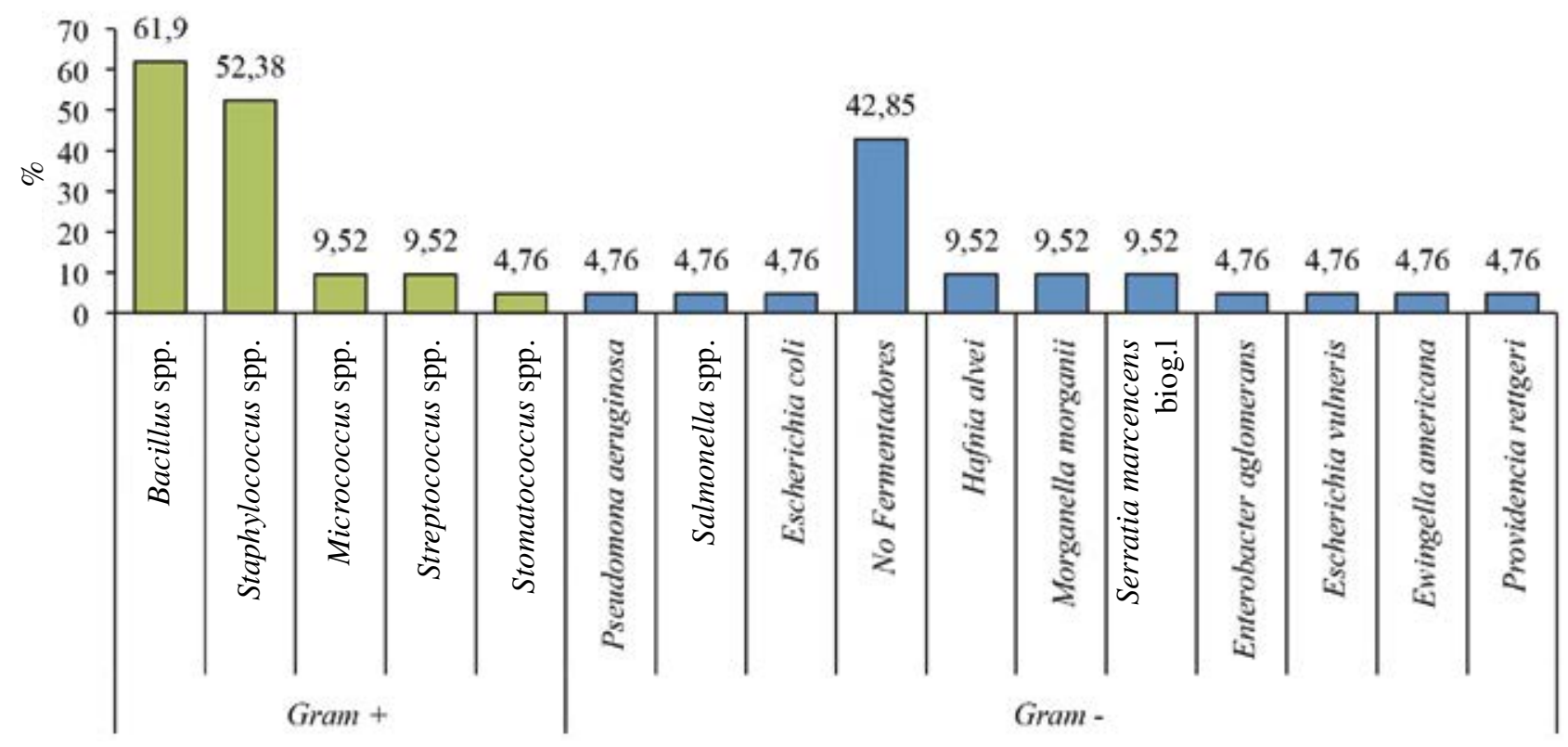

Figura1. Porcentaje de muestras de hormigas $(\mathrm{H})$ con diferentes cepas bacterianas aisladas. Percentage of ant samples $(\mathrm{H})$ with different bacterial strains isolated. 


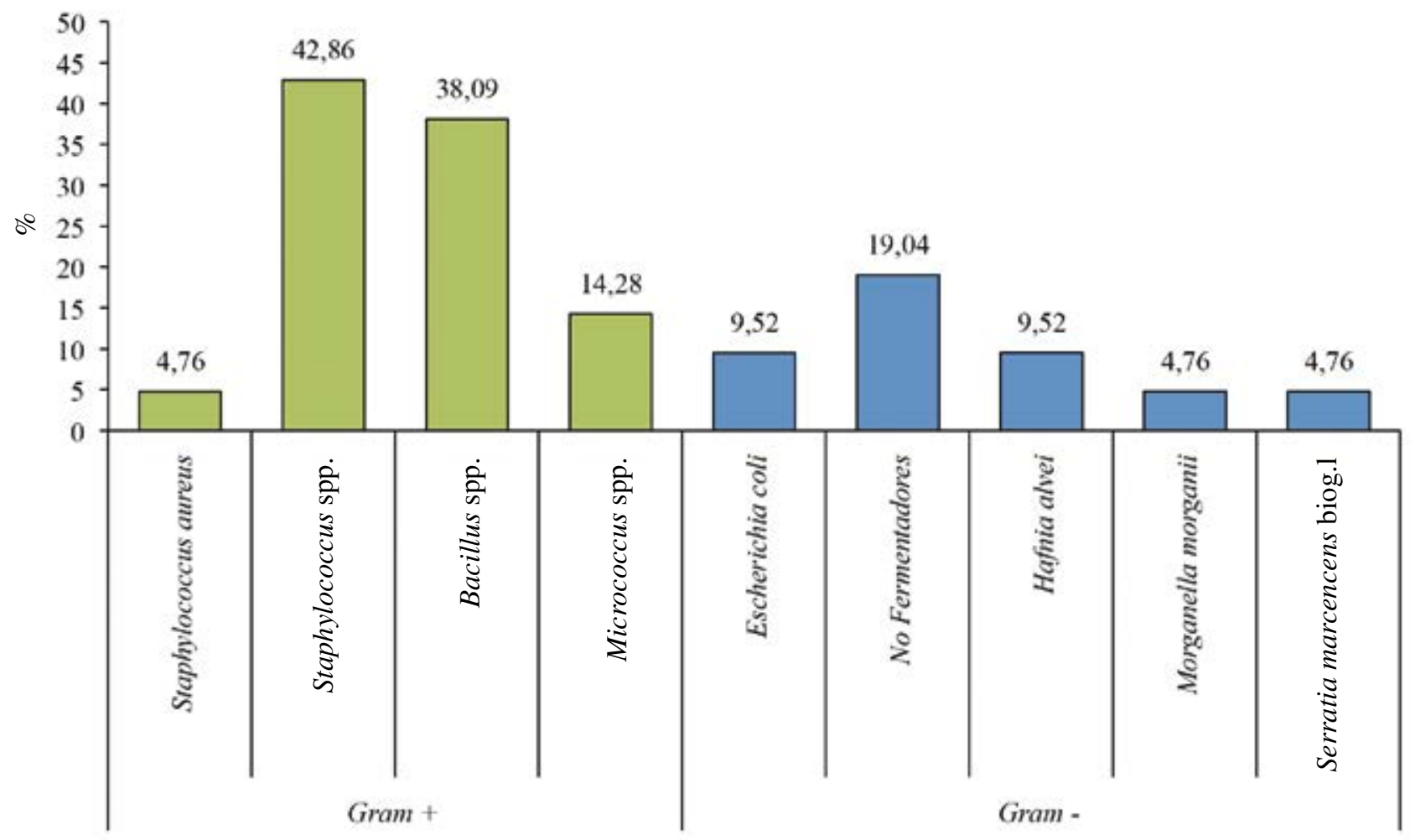

Figura 2. Número de muestras control B (expresado en porcentajes) en que se aíslan diferentes cepas bacterianas.

Control B Number of samples (expressed in percentajes) in which different bacterial strains are isolated.

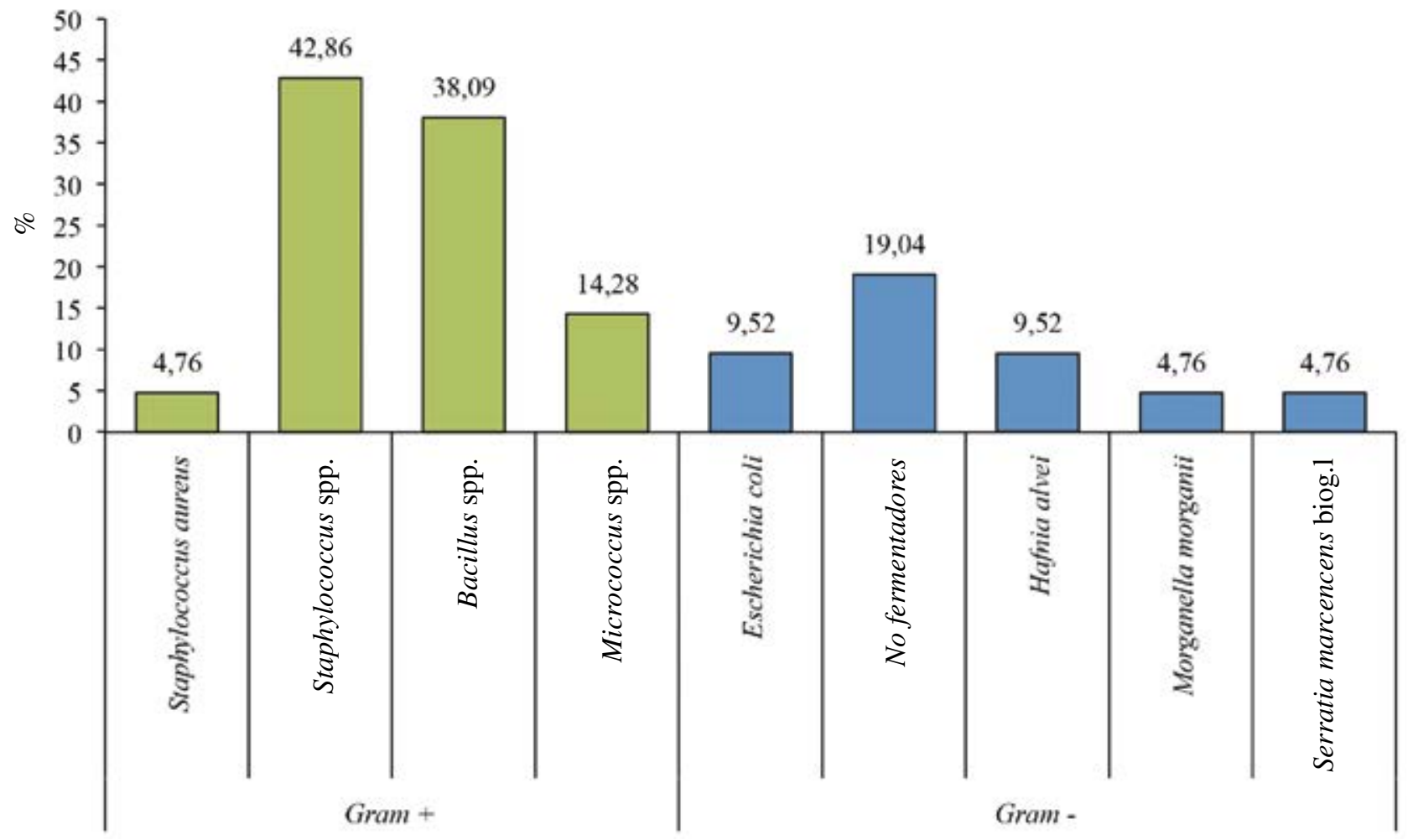

Figura 3. Frecuencias de presentación (expresado en porcentajes) de microorganismos en relación con el total de cepas aisladas en 21 muestras Controles B.

Presentation frequencies (expressed as a percentaje) of microorganisms in relation to total isolates in 21 samples Controls B. 
spp., seguido de Micrococcus spp. y Bacilos G- no fermentadores, con porcentajes menores de Clostridium spp. y Enterococos. Por último, con un porcentaje de $1,82 \%$ Enterobacter spp., E. aglomerans, Escherichia coli, Pseudomonas stutzeri y Difteroides, mientras que para las muestras posterior al paso de hormigas los mayores porcentajes fueron para Micrococcus spp., Bacillus spp. y Enterococos (Ipinza-Regla y col 1981).

Investigaciones realizadas por Ipinza-Regla y col (1984) indican la importancia de este formícido como vector de microorganismos patógenos en fábricas de hamburguesas y de confites. Considerando el aislamiento de Shigella flexneri como un hallazgo en este himenóptero como vector de dicho enteropatógeno, se le suma el hecho de que el riesgo potencial para la salud humana es mayor, porque basta un número reducido de células para causar infecciones sintomáticas.

En las fábricas de hamburguesas y de confites las frecuencias porcentuales más altas de aislamientos microbianos en muestras de hormigas la obtuvieron Streptococcus spp. y Enterobacter spp. (80\%) y Bacillus cereus $(66,6 \%)$, respectivamente. De ambas dependencias se obtuvieron muestras de hormigas que también arrojaron crecimiento microbiano de cepas que se aislaron en este trabajo. Algunas de ellas son Micrococcus spp., Streptococcus spp., Staphylococcus coagulasa (-), bacilos G- no fermentadores, Bacillus spp., E. coli, y $S$. aureus, entre otros.

En las muestras control B, las cepas que obtuvieron mayor porcentaje de aislamiento fueron Micrococccus spp., y Streptococcus spp. (fábrica de hamburguesas) y $S$. coagulasa (-) (fábrica de confites).

Resulta interesante comparar los resultados anteriores con aquellos obtenidos en este trabajo, ya que el patrón de aislamientos es similar.

Microorganismos del género Bacillus fueron los segundos que más frecuentemente se aislaron en las muestras de hormigas. No se logró identificar una especie en particular de este género, además, esta investigación orientó sus procedimientos a aislar e identificar mayormente seis microorganismos anteriormente señalados. Sin embargo, una vez que se aisló alguna cepa de Bacillus, a esta se le realizaron algunas pruebas de rutina para lograr identificar alguna de sus especies, especialmente B. cereus, siendo negativos para este caso.

Se han señalado, aunque poco frecuentemente, infecciones mamarias en cabras causadas por especies Bacillus (Blood y col 2002). No obstante, de todas las especies, Bacillus anthracis es el único agente patógeno importante.Otras especies se consideran comensales y patógenas en la mayoría de las circunstancias. Este género (Bacillus) se encuentra ampliamente distribuido en la naturaleza y la mayoría de las especies existentes se encuentran en el suelo, el agua, el polvo, el aire, las heces y en la vegetación.Las esporas son resistentes al calor y desinfectantes químicos.
Estudios en bovinos reportaron varios casos a $B$. cereus como un agente causante de mastitis agudas con graves consecuencias como necrosis de glándula mamaria, muertes espontáneas o un deterioro grave en el estado del animal, produciéndose la necesidad de sacrificarlos. La importancia de esto es que con el tratamiento adecuado los animales podrían recuperarse, sin embargo el daño que se produce a nivel mamario es alto, perdiendo su capacidad lechera, y por consiguiente convirtiéndose en un animal poco rentable (Shiefer1976).

En España, investigaciones acerca de mastitis clínicas en cabras han descrito a Bacillus spp., entre otros, como agentes causales en procesos de mastitis, sin embargo, en cuanto a presentación son menos importantes (Gallego y Pérez 1994).

Estudios realizados en Colombia (Albarracín y col 2008), demostraron una incidencia de Listeria monocytogenes de $3 \%$ y de Listeria spp. de $15 \%$ en leche cruda de cabra no pasteurizada, constituyendo un riesgo para la salud humana, considerando que el consumo de leche de cabra se ha incrementado en los últimos años debido a sus propiedades nutricionales, siendo los infantes y las personas mayores quienes más consumen esta leche. Para el aislamiento e identificación de este patógeno, tanto en los trabajos anteriores como en este, se siguió con el mismo procedimiento con la única diferencia de que el enriquecimiento selectivo se realizó en caldo Palcam en lugar del medio LEB.

Investigaciones realizadas por García y colaboradores 2009, en el estado de Zulia, en Venezuela, relacionadas con la calidad bacteriológica de la leche cruda de cabra, de muestras tomadas a nivel del pezón, arrojaron el hallazgo microbiológico de varios géneros, entre ellos se mencionan Staphylococcus spp. $(54,84 \%)$ (Staphylococcus coagulasa negativa 32,26\% y St. aureus 22,58\%), Streptococcus (22,58\%) (Streptococcus spp. $(19,35 \%)$ y St. agalactiae (3,23\%)), Micrococcus spp. (16,13\%) y Pseudomonas spp. $(6,45 \%)$. De acuerdo con los resultados, se concluye que en la leche de cabra producida en la parroquia Faría predominan especies asociadas a infección intramamaria, y elevados recuentos bacterianos, que evidencian una baja calidad bacteriológica de la leche, representando su uso en la elaboración de queso blanco fresco, a partir de la leche cruda, un riesgo para la salud pública.

Por lo anterior, resulta de gran interés conocer estos hallazgos, ya que la mayoría de estos agentes, coincidentemente, aparecen en los aislamientos de esta investigación. Lo que permite relacionar la importancia que tiene la hormiga argentina en su rol como vector de estos agentes, ya comprobado en esta investigación, y el riesgo sanitario tanto para el animal como para el hombre.

Dicho de otra forma, la mayoría de las especies que se han encontrado y aislado más frecuentemente en leche cruda de cabra coinciden con las mismas transportadas por la hormiga argentina en este trabajo, lo que sugiere que este formícido se comportaría como 
posible vector de estos agentes causantes de patologías mamarias en cabras.

Basados en los criterios microbiológicos definidos en el párrafo II, artículo 172 del Reglamento Sanitario de los Alimentos, se pudo identificar la clase de peligro que presentan los agentes microbianos encontrados. Es importante destacar que los agentes microbianos aislados en el presente estudio pueden considerarse desde microorganismos indicadores, de bajo, moderado y hasta grave peligro para la salud. Salmonella es uno de los microorganismos considerados para esta última clase mencionada anteriormente.

Los agentes microbianos que esta hormiga puede transportar tienen gran importancia en veterinaria. Staphylococcus spp. participa, en general, en procesos purulentos. S. aureus es el principal agente etiológico de las mastitis en bovinos, de la botriomicosis equina (infección granulomatosa con abscesos fistulosos del cordón espermático). En perros y gatos causa diferentes infecciones como abscesos, piodermas, otitis, conjuntivitis, mastitis.

De esta manera, si los planteles lecheros artesanales no toman las medidas sanitarias necesarias para el control de este vector, tanto en salas de ordeñas como en corrales, se podría considerar como un riesgo potencial para la salud del animal y contaminación de la leche.

\section{AGRADECIMIENTOS}

Los autores agradecen al Fondo de Investigación y Desarrollo Universidad Mayor (FIDUM); al Dr. Juan Burrow, Médico Veterinario asesor del plantel lechero de cabras en estudio ubicado en la comuna de Lampa, Región Metropolitana; a Miriam Troncoso, Tecnólogo Médico, por su colaboración en las técnicas de laboratorio; al Dr. Carlos Alvear, Médico Veterinario, por su asesoría en el análisis estadístico; y finalmente al Dr. Rafael Rodríguez, Médico Veterinario, e Isabel del Real, Ingeniero Agrónomo Mg Cs., por su colaboración en la confección de gráficos.

\section{REFERENCIAS}

Albarracín Y, R Poutou, A Carrascal 2008. Listeria spp y L. monocytogenes en leche cruda de cabra. Rev MVZ Córdoba 13, 1326-1332.
Blood DC, CC Gay, KW Hinchclife, OM Radostits. 2002.Tratado de las enfermedades del ganado bovino, ovino, porcino, caprino y equino. $9^{\mathrm{a}}$ ed. McGraw-Hill, Madrid, España.

Gallego L, J Pérez. 1994. Producción ovina y caprina. Compobell S.L., Murcia, España.

García U, J Rivero, P González. 2009. Calidad bacteriológica de la leche cruda de cabra producida en la parroquia Faría, municipio Miranda, estado Zulia, Venezuela. Rev Fac Agron 26, 59-77.

Ipinza-Regla J, G Figueroa, J Osorio. 1981. Iridomyrmex humilis "hormiga argentina" como vector de infecciones intrahospitalarias. I Estudio bacteriológico. Folia Entom Mex 50, 81-96.

Ipinza-Regla J, G Figueroa, J Moreno. 1984. Iridomyrmex humilis (Formicidae) y su papel como posible vector de contaminación microbiana en industrias de alimentos. Folia Entom Mex 62, 111-124.

Ipinza-Regla J, A Lucero, MA Morales. 1991. Hermetismo en sociedades de Camponotus morosus Smith, 1858 (Hymenoptera:Formicidae) en nidos artificiales. Rev Chile Entom 19, 29-38.

Ipinza-Regla J, I Castro, R Eissemann, MA Morales. 2010. Factors influencing the distribution of nests of the argentine ant (Linepithema humileMayr) (Hymenoptera:Formicidae), in foothills Ecosystem of central zone of Chile. Neotropical Entomology 39, 686-690.

Larraín P, J Ipinza-Regla, L Álvarez. 1995. Daño de la hormiga Solenopsis gayi (Spinola) (Hymenoptera: Formicidae) a mandarinos (Citrus reticulata Blanco) y pepino dulce (Solanum muricatum Ait) en la IV Región. Agricultura Técnica 55, 164-166.

Lazo QJ. 2002. Experiencia con un plantel productor de carne caprina en la Región Metropolitana. Tecno Vet 8.

Olaya L, P Chacón de Ulloa, A Payan. 2005. Ants (Hymenoptera:Formicidae) in hospital center of valle del cauca as vectors of nosocomial pathogens. Rev Colomb Entomol 31, 183-187.

Osorio R 2010. Efecto de la depredación de tres formícidos chilenos frente a Reticulitermes flavipes (Kollar 1837) (Isoptera:Rhinotermitidae). Tesis, Facultad de Ciencias Silvoagropecuarias, Universidad Mayor, Santiago, Chile.

Ripa R, F Rodríguez, S Rojas. 1993ª . Nuevos avances en el manejo del chanchito blanco de la vid. Investigación y progreso agropecuario. La platina 76, 28-30.

Ripa R, F Rodríguez, S Rojas.1993 . Plagas más importantes de los cítricos y su manejo II.- Ácaro de la yema, escama roja, conchuela acanalada, pulgones, caracol de las viñas, control de hormigas, manejo de la poda. Investigación y progreso agropecuario. La Platina 79, 5-11.

Ripa R, F Rodríguez, M Rust. 1998. Las hormigas y su relación con la agricultura. Rev Fruticola 19, 85-92.

Schiefer B. 1976. Pathology of Bacillus cereus mastitis in dairy cows. Can Vet J 17, 239-243. 
